\title{
A Novel Technique for Region-Based Features Similarity for Content-Based Image Retrieval
}

\author{
IMRAN MEMON*, QASIM ALI ARAIN**†, AND NASRULLAH PIRZADA*** \\ RECEIVED ON 14.11.2016 ACCEPTED ON 13.11.2017
}

\begin{abstract}
The study of feature extraction and clustering techniques for an image is carried out to find the future scope in the area of image clustering for the real world applications. Nowadays, a huge amount of image data is being collected in various application domains. As clustering approaches group homogeneous data together and also deals with unlabeled data, it is used quite often for accessing the interesting data easily and quickly. Image clustering is a process of grouping image data on the basis of similarities present between them. Features extracted from the images are used for the computation of similarities among them. This paper presents a technique for CBIR (Content Based Image Retrieval) by selecting the regions on the basis of their contribution to image contents. Texture and edge features are extracted at region-level whereas shape feature is extracted at image-level. At region-level image is divided in nonoverlapping regions. Texture and edge features are calculated for each region separately. Curvelet transform is used for extracting the texture feature by providing the curve continuity as well as line continuity in the feature extraction process. Moment invariant is used for extracting the shape features. All of the regions of the image may not have equal contribution in identifying the user perception of the image. Proposed method does not dominate to the non-highlighted regions but it decreases the region weight for less contributing regions. IRM (Integrated Region Matching) technique is used for retrieving the relevant images. The performance of the proposed system is tested on Flickr and COREL databases. Experimental results show that the retrieval performance of the proposed algorithm is better in comparison to other state-of-the-art methods.
\end{abstract}

Key Words: Region Matching, Content Based Image Retrieval, Similarity, Localization

\section{INTRODUCTION}

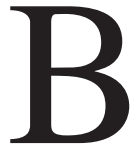

$y$ the increase in multimedia contents over the web throws a challenge to manage and retrieve information from the heterogeneous collection of data. Initially text based approaches were used by various researchers, but rapid growth in multimedia contents made it inefficient, due to the need of annotation. More advancement in this field leads toward the retrieving images on the basis of their contents. Retrieval of the images on the basis of their features such as color, texture and shape is termed as CBIR. The feature extraction level

$\dagger$ Corresponding Author (E-Mail: qasim_ali_arain@yahoo.com)

* College of Computer Science, Zhejiang University, Hangzhou 310027, China.

** Department of Software Engineering Mehran University of Engineering \& Technology, Jamshoro

*** Department of Telecommunication Engineering, Mehran University of Engineering \& Technology, Jamshoro.

This is an open access article published by Mehran University Research Journal of Engineering and Technology, Jamshoro under the CC by 4.0 International License. 
measured by image contents level such as primary level is known as low level, however, the features extracted based on color, shape, texture, spatial information are called primitive features. The pixel information can be obtained by extracting pixel representation of the images. Indexing and retrieval are two essential issues to be addressed in visual information systems and CBIR, which has been considered as a powerful approaches for accessing visual information systems, which may allow users to search for the desired images of their true content with the aid of the technique in image processing and computer vision. Content-based image indexing and retrieval have attracted greater attention for the last few decades.

The demand for development of CBIR system is improved due to enormous increase in amount of images and widespread applications in multiple fields as well. Colorization is an art of adding color to gray images or movies. This problem was firstly considered by Fornasier, who insists on recovering paintings in an Italian church which was destroyed in World WarII. A major difficulty of colorization lies in the fact that colorization may not have the "correct" answers since the gray image only has one dimensional (luminance or intensity) while the color image involves 3D (Three Dimensional) pixel values. Hence, human interaction may play an important role in the colorization process which is time-consuming and expensive. In recent years, various methods are proposed to solve this problem which are mainly based on: color transfer, motion estimation, color prediction, probabilistic relaxation, segmentation, chrominance blending and variational model based methods. In this paper, we focus on variational model based methods. In [1] present a colorization method which may use the morphological distance transformation and image structures to propagate color within the gray scale image. In [2] propose an image colorization algorithm based on norm minimization of the mixed 10/11, and they assume that the difference of color values between neighbor pixels is given as a monotonically increasing function of the difference of gray values between neighbor pixels. Moreover,they propose a colorization method by constructing matrix with high compression efficiency and using optimization method for the colorization-based coding. However they formulate the colorization-based compression problem into an optimization problem by constructing the colorization matrix in a multiscale manner. In [3], researcher propose a hybrid scheme that combines both the example-based colorization and the scribblebased colorization algorithms. Moreover they present a colorization method in YIQ color space which requires neither region tracking nor precise image segmentation. Their basic assumption is that neighboring pixels in an image with the similar intensities should have the similar color. This method can be used not only in a still image but also as an image sequences. It is an effective interactive colorization method in many cases. In [4], researcher propose two variation models for image colorization in CB (Chromaticity and Brightness) color space. The chromaticity component lives on the 2D sphere.

Various researchers contribute their precious efforts in the field of CBIR [1]. CBIR can be applied for both texture and non-texture images. Methods [1,2] based on CBIR for texture image retrieval, might be different from nontexture image retrieval. Enhancement to the CBIR continue to ROI (Region of Interest) [3] based image retrieval. This approach provides better user interaction 
with the system. Retrieving images from the large databases in optimum time and with the higher efficiency has always been the issue of concern. Performance of image retrieval is solely depends upon the feature extraction process. Low level features do not deal well with the human perception of the image. Therefore image retrieval system requires the efficient use of these features. High level semantics are developed to reduce the semantic gap of user and system understanding. There have been various retrieval systems designed and implemented for achieving optimal performance of retrieval system. Some approaches [5] used the concept of relevance feedback for improving the retrieval performance and user interaction with the system as well. Image segmentation may also improves the retrieval performance of the system.

Our contribution to this research might be explained as follows:

(1) We proposed image feature extraction method, that is performed by giving more priority to the areas having high variations in the pixel and reduce the burden of the user for selecting the ROIs.

(2) We proposed novel approach for various features such as color, shape, size and text from the integral images. It performs better and provides more accuracy in terms of texture feature at the curves.

(3) We compare our proposed method with existing methods by using Flickr database images and [1-4] from corel-1000 database, simulation results has revealed that our proposed method is more accurate, fast and robust.

\section{RELATED WORK}

Fourier transform has been proved very poor in the representation of discontinuous objects. Curvelet provides better edge representation due to the decomposition of image at different scales and orientations. This provides better directional representation than the other wavelet variants. Implemented initially with the curvelet transform. This implementation leads with the poor speed of feature extraction. Wrapping method proposed are used with curvelet to minimize the feature extraction time with the same effect of features. It suggests the selection of most relevant feature set among different features by providing the feature discrimination technique [6]. This decreases the retrieval time of the system. Genetic algorithm is used for selecting the optimal boundaries of the feature set hence it reduces the complexity of the feature set. Proposed image retrieval technique on the basis of motif co-occurrence matrix and weight vector associated with it.However, due to the smaller size, it does not provide the calculation with the boundaries of the block and results in information loss. Variant of standard LBP (Local Binary Pattern) is proposed. This descriptor exhibits the directional property with the binary pattern [7]. Inclusion of directional relation of the pixel with neighboring pixel improves the performance of image retrieval. Single pixel is analyzed in four different directions with its neighbors. Since this approach depends upon the central pixel. Noise may affect the value of DLEP code. Images are retrieved in a feed forward manner in the method proposed.They perform the reduction in the search space by reducing the database at each level on the basis of the output produced by the previous stage. However, this progressive approach provides more weight to the color 
feature than shape and texture.Therefore, this method does not perform well on heterogeneous databases. To provide the more interaction with the user, ROI based image retrieval method is proposed.Region codes are assigned to each of the region with the highest priority is given to the center region. This method suffers from dependency of the target ROI with the queried ROI. Location dependency of ROI is removed [4] .They proposed a region based image retrieval system that is independent to the location of the region. Instead of location they prefer the property of the region. Regions with the similar property are compared with each other. This approach performs the image retrieval by applying the blocks of different sizes for image segmentation [810]. Generally, traditional CBIR based approaches performed feature extraction for the complete image by providing equal opportunity to all intensity values. This will lead to some irrelevant images in retrieval process. To overcome this issue, it is been used the concept of region which is extracted on the basis of highest attractiveness. These extracted region works as system defined ROI. However, in [11] some regions dominate the other regions completely due to the alike attractiveness. They provided higher weight to the center region only whereas applied weights to the features of the image to address the issue of irrelevant images.

Recent report work shows that text of images like their captions, tags and their descriptions have been used as extra features for good understanding and illustration of images.The text around images may considered as feeble supervision, side information, meta-information, may describe a detailed semantics that will elaborate object back ground and events related with images. To use multimodal description will add information which will help to elevate the retrieval and indexing throughput of images. Recently report work related to multimodal image indexing and retrieval is generally describe techniques that would identify current feature space for the image representation by combining features like LSI, (pLSA) and NMF [1], or we may say the connection between multimodal structures in order to produce a new illustration for every image .Nevertheless certain shortcomings to these approaches have been discovered. It has been observed that, these approaches cannot perform online learning, That's why it is difficult to handle a live stream of images that require continuous updates. The text surrounded by the images may have descriptive keywords and the quantity is large enough so that it will be very difficult to describe the image contents, however such erroneous information may cause certain side effects in the derived feature space. This may result in a sluggish convergence time when the data set is huge.

OMC-ART used to employ multimodal search by applying graphical structures, keywords or fusion of both, moreover it will apply a ranking algorithm to select optimized cluster in abstraction layer and henceforth to apply sorting images in the ranked list. This ranking algorithm is going to reduce application cost due to already designed ranking of clusters, this technique is more effective when partial amount of images to be recovered. Performance evaluation of OMC-ART with help of published web images i.e. NUS-WIDE and Corel5K data set has been performed. During the analysis, multiple aspects have been considered for the evaluation; they might be reclamation performance evaluation and proficiency analysis. It is obvious from investigations of outcomes that OMC-ART has outperformed in terms of mean average precision, 
precision and recall and it also has much efficient response time. There are two approaches to be followed during multimodal image indexing and retrieval. During,first approach an existing algorithm to be extended for image indexing by using a single type of features for integrating multiple types of features[12]; moreover these collections of images can be collected from different web resources like flicker and picassa. Therefore, the top strategy to be considered for combining multimodal information can be described as: Late fusion and early fusion. The first one is also identified as rank accretion at decision level which comprises of the processing step of each data source separately during the indexing phase. The previous work [8] is an example of similarity combination to attain multimodal access in image acquisition while on the other hand, early fusion technique will help to model the feature relationship to design a new multimodal illustration during decision phase, it is only been required to analyze the multimodal features [9-10]. We have categorized our work as an initial fusion technique for multimodal image analysis. Recently topic analysis is used to describe the connection among multimodal data that would be specific to images and text comments. Nevertheless, the recent techniques may fail at exploiting accessibility of multimodal information for effectually demonstrating content of images. The current trends are not competent enough and fail at manipulating the availability of multimodal information to effectually symbolize the content of images. Recently research has done a lot of development in the field of AIA (Automatic Image Annotation) to overcome the shortcomings of content based image retrieval. The main purpose of AIA technique is to assure images with labels, user may search for images by using certain keywords.
Oracle in image search: A content-based approach to performance prediction;

In this domain the prediction of Query Scenario is a hot topic in information retrieval and hence forth it is very important for many applications like query suggestions met search and query expansions. We can define two approaches, one can be managed by examining query also data gathering and second one is by computing search results. Thereafter they are being called as pre and post search techniques. There are many mechanisms have been established for the searching of text domain however extra detail will be presented in the later sections. Moreover, there is still a lot of areas that are not been addressed on performance expectation for multimodal search based on text queries. There are still many research methods which may be applied to multimedia search problems, if comparison would be made with the post search approaches, we can find pre search methods are unable to achieve the desired performance because there is no evidence about search results. However, it obvious from discussion that post search method may not be applied to multimedia search as text query also image search results belongs to dissimilar media types. Moreover there is a technique to interchange the multimedia entity by its textual information which might contain it is title and text information. This can be usually done by technique called indexing multimedia objects in commercial search engine. By reviewing the literature it can be deduced that there is a much cavity among the text narrative and information of the multimedia object [13-14] .Therefore, post search techniques will also not work on multimodal search. We have given an image hunt performance prediction technique by doing experiments on visual content of pursuit outcomes. During this scenario a text query and an image ranking list without 
labeling would be considered. Task is to envisage search performance by considering certain assessment metrics that might be AP (Average Precision) or NDCG (Normalized Discounted Cumulative Gain). During this technique, we have analyzed AP and NDCG and then concluded that we may need to calculate images relevance probabilities to calculate mathematical anticipations. We have given an approach called query adaptive graph based learning to complete task, during this multiple image representation may use according to query type resolute by query classification component. To explain statistics related to inverse term and document frequency. It has been deduced that better reclamation performance is directly proportional to t high variation of inverse document frequency of query term. Other external sources like segmentation may employ to measure the association between query terms. It has been stated that the query term that would always appear in the same or related sense might be considered ambiguous and hence it is very much anticipated to accomplish best. These techniques are much faster as they don't need to apply for a search operation; the performance might not be according to the desired search results. They may not be taken into consideration.

Harvesting visual concepts for image search with complex queries:

Recent studies have shown that a significant number of research efforts have been made to improve stretched query presentation in text based information repossession. These might categorize into instinctive term re-weighting $[4,16]$ and query lessening $[5,15-16]$ approaches. However it has been proved that by allocating suitable weight to query ideas has a very positive effect on retrieval performance [7]. They [4] have designed and assessed an algorithm that would assign weights to recognized main concepts in verbose query, and also shown enhanced reclamation efficiency. It has given a novel regression structure approach to calculate terms depend upon information from previous queries. Another novel approach beyond unsupervised calculation of concepts was given in [4], which is going to calculate weight of query by using parameterized combination of assorted importance feature. A new and important idea has been given by researcher in which cropping complex query to remain only with the important term is also known as one of the important side to improve hunt throughput.They have given another idea of interactive query induction in this approach user presented by the topmost 10 graded queries beside with corresponding topmost classification snippets. Moreover, a lot of study exertions have been accompanied by designing multifarious queries in media search. However literature related with complex queries still has to be very vague. The current techniques may interpretation the query term autonomously or require rigorous human collaboration [17-19]

\section{THE PROPOSED METHOD}

Similarity measure is an important part of the clustering process shown in Fig.1. Prior to similarity measure, features of the selected image need to be extracted. Image features are of two types, low level features such as color, texture and shape or high level features like semantic. With the help of extracted features, similarity between images is computed. Some of the popular image feature extraction methods are discussed below.

\subsection{Features and Similarities}

Color: Color is a basic but most expressive feature of the image. This feature is robust because it is not more dependent on size, orientation and viewpoint of the image. Selection of appropriate color space is the first stage of 
color feature extraction. Color space is a mathematical model which represents colors. In year 1931, CIE (Comission Internationale de l'Eclairage (International Commission on Illumination)) introduced XYZ (Tristimulus values) color space. RGB (Red, Green, Blue), HSV (Hue, Saturation, Value), CMYK (Cyan, Magenta, Yellow, Key (Black)), YUV (Y-luminance, U and VChrominance are the most popular color spaces. CIE $\mathrm{L} * \mathrm{u} * \mathrm{v}$ (L*-luminosity, $\mathrm{u}^{*} \mathrm{v}^{*}$-chromaticity coordinates) and CIE L*a*b* (L*-luminosity, a*-red/green axis, b*yellow/blue axis) are also introduced by CIE. RGB is an additive approach so all possible colors can be created from red, green or blue. RGB is not perceptually uniform color space. But HSV, CIE L* $\mathrm{u}^{*} \mathrm{v} *$ and CIE L*a*b* are perceptually uniform color spaces, which are proportional to the human perception. HI (Histogram Intersection), DC (Dominant Color) and CC (Color Correlogram) are most common color-based image descriptors which are discussed as follows:

$\bullet$

HI: Considers global color features with the help of color histograms. Low computational cost, robust against geometrical modifications such as rotation, resolution and scaling
DC: Represents the image by using a small number of dominant colors

- $\quad$ CC: Considers global as well as local distribution of each color in the image

They devised a new color descriptor which is the combination of DC color descriptor and CC descriptor. Global distribution of colors in the image is considered by DC. Whereas local color distribution is taken into account by the CC. This method considers both, global as well as local color distribution. Proposed descriptor is faster than CC descriptor.

Color similarity is measured with the help of color moments. Mean, median, variance, standard deviation, skewness and kurtosis are some of the popular color moments. Color feature extraction techniques used for the image retrieval. In HSV color space, binary Haar color descriptor outputs better results. PCMD and CHMD provide good results in the CIE $\mathrm{L}^{*} \mathrm{u}^{*} \mathrm{v}^{*}$ and the CIE $\mathrm{L} \mathrm{a}^{*} \mathrm{~b} *$ respectively. Only color features may not be helpful to get the accurate results for many applications. Hence, texture features need to be combined with the color features to improve efficiency. Some more image

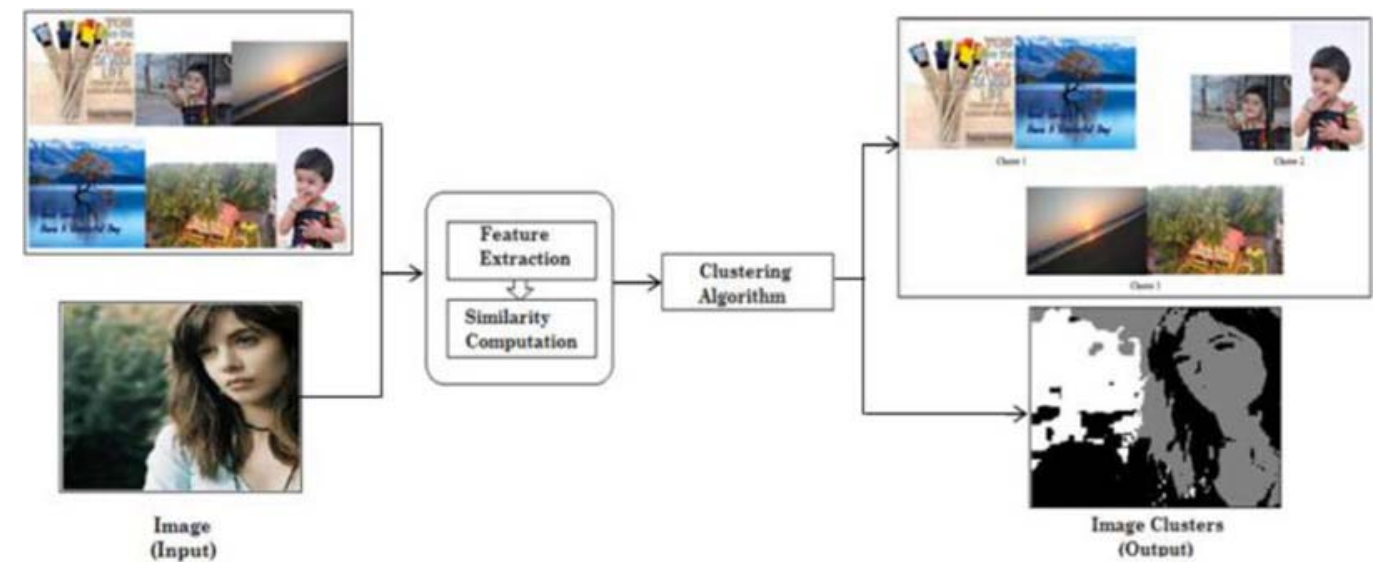

FIG. 1. REGION SEMINARIES IMAGE BASED MODEL 
feature extraction approaches are discussed below: Quad Histogram-based color features extraction method is developed with the help of quad-tree to specify the homogenous blocks of different sizes. Quad Histogram is an efficient technique as compare to Color histogram and histogram is powered by the complexity of image .To improve performance of the descriptors, combine color moment and local features with the help of SIFTscale-invariant feature transform. Generalized color moment invariants (by analyzing shadow-shading quasiinvariants) are used to construct the color invariant regions.

\subsection{Texture Feature Extraction}

Texture is a spatial property of the image. To extract texture feature, various approaches such as wavelet transform, Gabor filter, Laws texture energy, gray level co-occurrence matrix are available which are divided into following categories:

(1) Statistical approach

(2) Structural approach

(3) Spectral approach

Statistical approach characterizes stochastic properties of the spatial distribution of grey levels in the image. It is a quantitative measure of the arrangement of intensities. GLCM (Gray Level Co-Occurrence Matrix) and CCM (Color Co-Occurrence Matrix) are statistical approaches which are commonly used to extract the texture feature and find the spatial dependencies. In GLCM, texture is represented as matrix by using grey levels from the image. It implies the roughness and repetition of the texture in image. It is able to distil the character quantity of texture such as contrast, energy, entropy, local homogeneity, cluster shade, cluster prominence, maximum probability, etc. Statistical approach is easy to implement as compared to other approaches. By using GLRLM (Gray Level Run Length Matrix), local relative texture features are extracted in the form of global image properties. This approach is used for the extraction of higher order statistical texture features. GLRLM is a two-dimensional matrix generated by using grey levels and longest run. Each element of the matrix gives total number of occurrences of runs with a particular length of grey level in the given direction. Local relative GLRLM is compared with traditional GLRLM where it is found that Local relative GLRLM performs better. It is useful for embedding the local information in global feature extraction method.

\subsection{Shape Feature Extraction}

Shape is also a basic and important feature which can be used to describe the image contents. In this subsection some of the shape feature extraction techniques are discussed. Shapes can be described with the help of salient points, which are also referred as saliences or corners. It represents a shape in compact manner and it is invariant to the rotation and translation. These points are having high curvature .Similarity measurement process is given as follows:

(1) Detection of the salient points

(2) Representation in the form of feature vector

(3) Measure the similarity

As in the earlier technique salient points are used to describe the shapes, have used the edges for extraction of shape feature. Those edges are detected using SUSAN (Smallest Univalue Segment Assimilating Nucleus) operator. SUSAN operator is edge and corner 
detector which works on the basis of local area brightness. Further, moment invariants of the edge map are calculated which is used as a shape feature vector. This technique is simple and robust against noise. Here, moment invariants are of a high stability which gives very good description of the shape feature. A common Fourier descriptor method where Fourier descriptors with brightness are used for the shape feature extraction. By using Fourier transform for shape signature, Fourier coefficients and standardized pixel brightness are computed. Fourier coefficients are invariant to translation, scaling and rotation. Shape feature vector is made up of Fourier descriptors. Change of the start point is used as Fourier descriptor. Centroid distance function is used to compute the shape signature from boundary pixels of shape. Systems with this technique give better performance. This technique accomplishes boundary pixels computation using edge detector such as canny edge detector shape signature computation using boundary pixels and Fourier descriptor computation. Integral kernels are also used for shape description. Distinctiveness of the local shape geometry is a given by series of isotropic kernels which provides invariance properties. It is characterized at multiple scales which forms a signature and used as a compact description of the shape features. This signature is invariant to the group transformation such as translation, rotation, scaling, and reflection. Range of various kernel sizes is employed. This method is also robust against noise. Distance between two shapes is calculated based on their shape signatures using Wasserstein distance. Group transformation invariance can be extended by considering kernel with different characteristics. Affine invariant shape descriptor can be developed by modifying the characteristic kernel as anisotropic.

\section{EXPERIMENTAL RESULTS}

This section provides the detailed description of the experimental aspects in Table 1 covered by the proposed approach. Proposed image retrieval system is implemented in MATLAB. Flickr [20] and COREL [12,13] databases are used for experimental and comparison purposes as the benchmark databases shown in Tables 2-4. Retrieval results for example query images are shown in Fig. 2. The average retrieval time (in seconds) for the proposed curvelet-based dataset- 1 and dataset- 2 are shown in Fig. 3. Retrieved images for sample query images from each category represented in Fig. 4.

TABLE 1. VARIOUS IMAGE CATEGORIES

\begin{tabular}{|c|c|}
\hline Category ID & Image Category \\
\hline 1 & Dinosaur \\
\hline 2 & Beach \\
\hline 3 & Mountain \\
\hline 4 & Bus \\
\hline 5 & Horse \\
\hline 6 & Elephant \\
\hline 7 & Flower \\
\hline 8 & Building \\
\hline 9 & Africans \\
\hline 10 & Food \\
\hline
\end{tabular}

Mehran University Research Journal of Engineering \& Technology, Volume 37, No. 2, April, 2018 [p-ISSN: 0254-7821, e-ISSN: 2413-7219] 
A Novel Technique for Region-Based Features Similarity for Content-Based Image Retrieval

TABLE 2. VARIOUS IMAGE CATEGORIES VS AVERAGE AND RELATIVE TIME

\begin{tabular}{|c|c|c|}
\hline Descriptor & Average & Relative \\
\hline \multicolumn{3}{|c|}{ Extraction Times (milliseconds) } \\
\hline Dinosaur & $03+0.9$ & 0.81 \\
\hline Beach & $0.4+1.1$ & 1.00 \\
\hline Mountain & $0.6+0.9$ & 1.45 \\
\hline Bus & $1.1+1.3$ & 2.69 \\
\hline Horse & $2.3+3.0$ & 5.58 \\
\hline Elephant & $2.4+0.9$ & 6.05 \\
\hline Flower & $2.6+1.3$ & 6.31 \\
\hline Building & $3.3+0.9$ & 8.18 \\
\hline Africans & $15.0+4.3$ & 37.13 \\
\hline Food & $15.5+8.8$ & 40.89 \\
\hline \multicolumn{3}{|c|}{ Distance Times (microseconds) } \\
\hline Dinosaur & $28.6+7.5$ & 0.65 \\
\hline Beach & $29.9+1.4$ & 0.58 \\
\hline Mountain & $32.0+1.4$ & 0.73 \\
\hline Bus & $36.4+2.9$ & 0.83 \\
\hline Horse & $43.2+2.0$ & 0.98 \\
\hline Elephant & $43.8+1.7$ & 1.00 \\
\hline Flower & $51.4+1.6$ & 1.17 \\
\hline Building & $52.1+1.8$ & 1.19 \\
\hline Africans & $60.7+2.0$ & 1.38 \\
\hline Food & $71.8+7.1$ & 1.64 \\
\hline \multicolumn{3}{|c|}{ Feature Vector Sizes } \\
\hline Dinosaur & 7.875 & 0.03 \\
\hline Beach & 15.875 & 0.06 \\
\hline Mountain & 48.00 & 0.19 \\
\hline Bus & 61.50 & 0.24 \\
\hline Horse & 256.00 & 1.00 \\
\hline Elephant & 256.00 & 1.00 \\
\hline Flower & 512.00 & 2.00 \\
\hline Building & 512.00 & 2.00 \\
\hline Africans & 736.00 & 2.88 \\
\hline Food & 864.00 & 3.38 \\
\hline
\end{tabular}

Mehran University Research Journal of Engineering \& Technology, Volume 37, No. 2, April, 2018 [p-ISSN: 0254-7821, e-ISSN: 2413-7219] 
A Novel Technique for Region-Based Features Similarity for Content-Based Image Retrieval

TABLE 3. AVERAGE PRECISION ON TOP 10 IMAGES ON COREL DATABASE

\begin{tabular}{|c|c|c|c|c|c|c|}
\hline No. & Category & 1 & 2 & 3 & 4 & Proposed Work \\
\hline 1. & Africans & 72.8 & 73.6 & 74.8 & 69.7 & 74.4 \\
\hline 2. & Beaches & 89.0 & 46.3 & 58.2 & 54.2 & 87.1 \\
\hline 3. & Building & 70.3 & 74.3 & 62.1 & 63.9 & 75.6 \\
\hline 4. & Buses & 83.9 & 92.2 & 80.2 & 63.9 & 75.6 \\
\hline 5. & Dinosaurs & 100 & 99.6 & 100 & 98.7 & 100 \\
\hline 6. & Elephants & 69.8 & 54.1 & 75.1 & 48.8 & 72.2 \\
\hline 7. & Flowers & 92.7 & 92 & 92.3 & 92.3 & 95.7 \\
\hline 8. & Flowers & 92.7 & 92 & 89.6 & 89.45 & 90.2 \\
\hline 9. & Mountains & 71.6 & 50 & 56.1 & 47.3 & 88.3 \\
\hline 10. & Food & 57.2 & 82.7 & 80.3 & 70.49 & 70.4 \\
\hline \multicolumn{2}{|c|}{ Average } & 79.39 & 75.91 & 76.87 & 72.44 & 83.87 \\
\hline
\end{tabular}

TABLE 4. AVERAGE PRECISION ON TOP10 IMAGES ON FLICKR DATABASES

\begin{tabular}{|c|c|c|c|c|c|c|}
\hline No. & Category & 1 & 2 & 3 & 4 & Proposed Work \\
\hline 1. & Rose & 52.1 & 76.2 & 56.8 & 78.7 & 82.4 \\
\hline 2. & African & 43.2 & 70.5 & 39.5 & 69.9 & 79.2 \\
\hline 3. & Bird & 36.5 & 81.6 & 46.4 & 87.9 & 87.7 \\
\hline 4. & Cat & 86.1 & 92.1 & 82.6 & 94.2 & 91.4 \\
\hline 5. & building & 82.1 & 91.8 & 78.2 & 93.6 & 90.1 \\
\hline 6. & Automobile & 43.5 & 85.4 & 48.2 & 87.7 & 88.5 \\
\hline 7. & Frog & 69.2 & 76.8 & 71.9 & 78.4 & 79.7 \\
\hline 8. & Horses & 69.2 & 89.3 & 73.6 & 95.1 & 88.4 \\
\hline 9. & Ship & 42.5 & 75.4 & 56.8 & 79.7 & 81.5 \\
\hline 10. & water & 64.2 & 82.6 & 68.9 & 84.1 & 84.2 \\
\hline \multicolumn{2}{|c|}{ Average } & 58.86 & 82.1 & 59 & 82.88 & 85.31 \\
\hline
\end{tabular}

Mehran University Research Journal of Engineering \& Technology, Volume 37, No. 2, April, 2018 [p-ISSN: 0254-7821, e-ISSN: 2413-7219] 


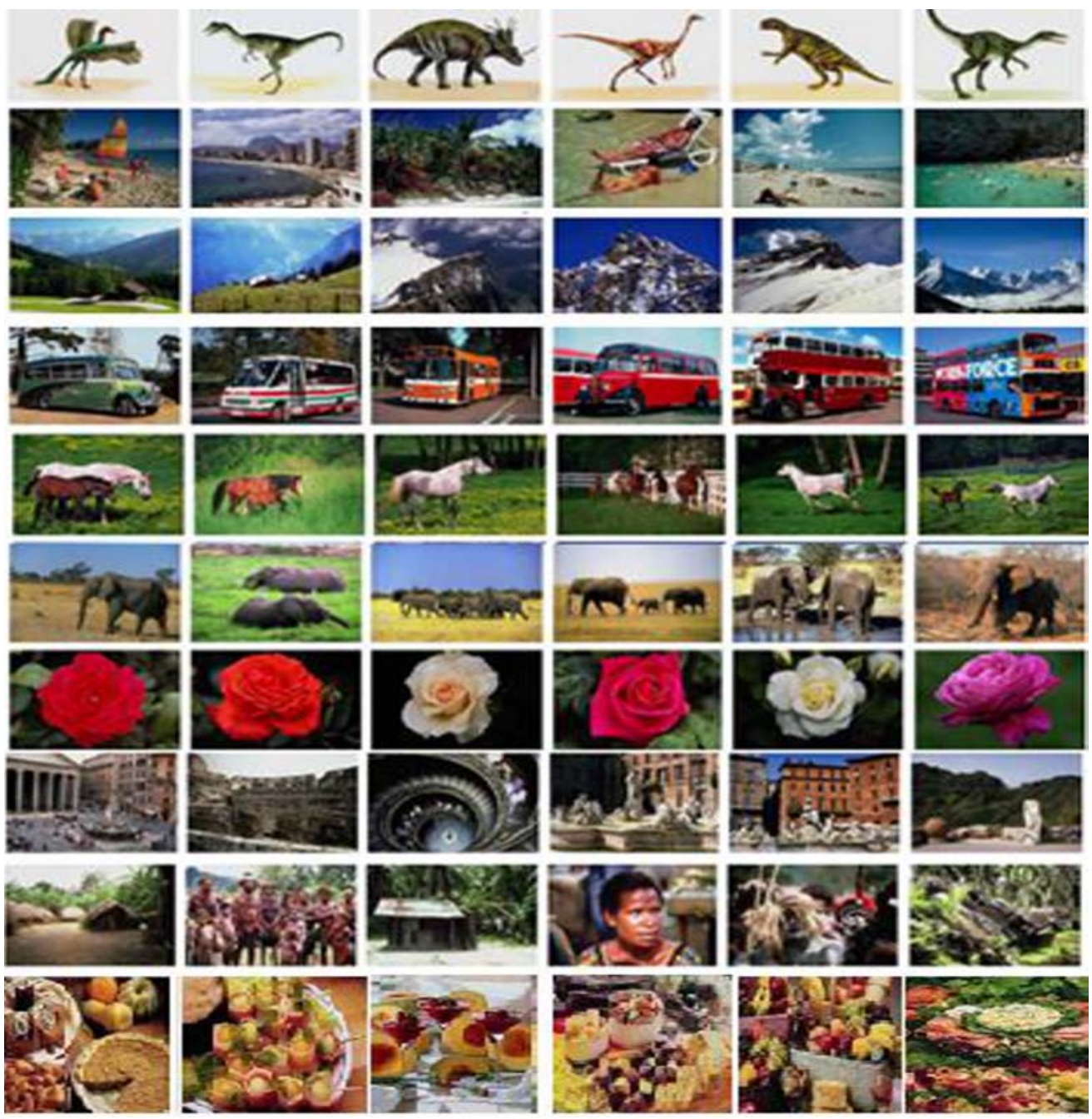

FIG. 2. RETRIEVAL RESULTS FOR EXAMPLE QUERY IMAGES

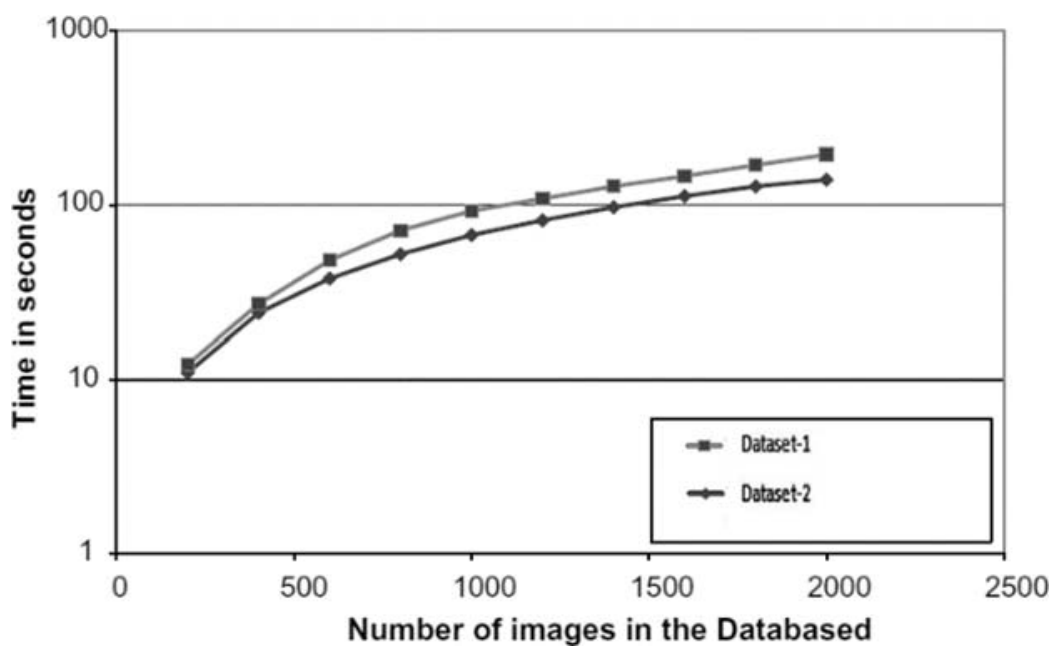

FIG. 3. THE AVERAGE RETRIEVAL TIME (IN SECONDS) FOR THE PROPOSED CURVELET-BASED DATASET-1 AND DATASET-2 


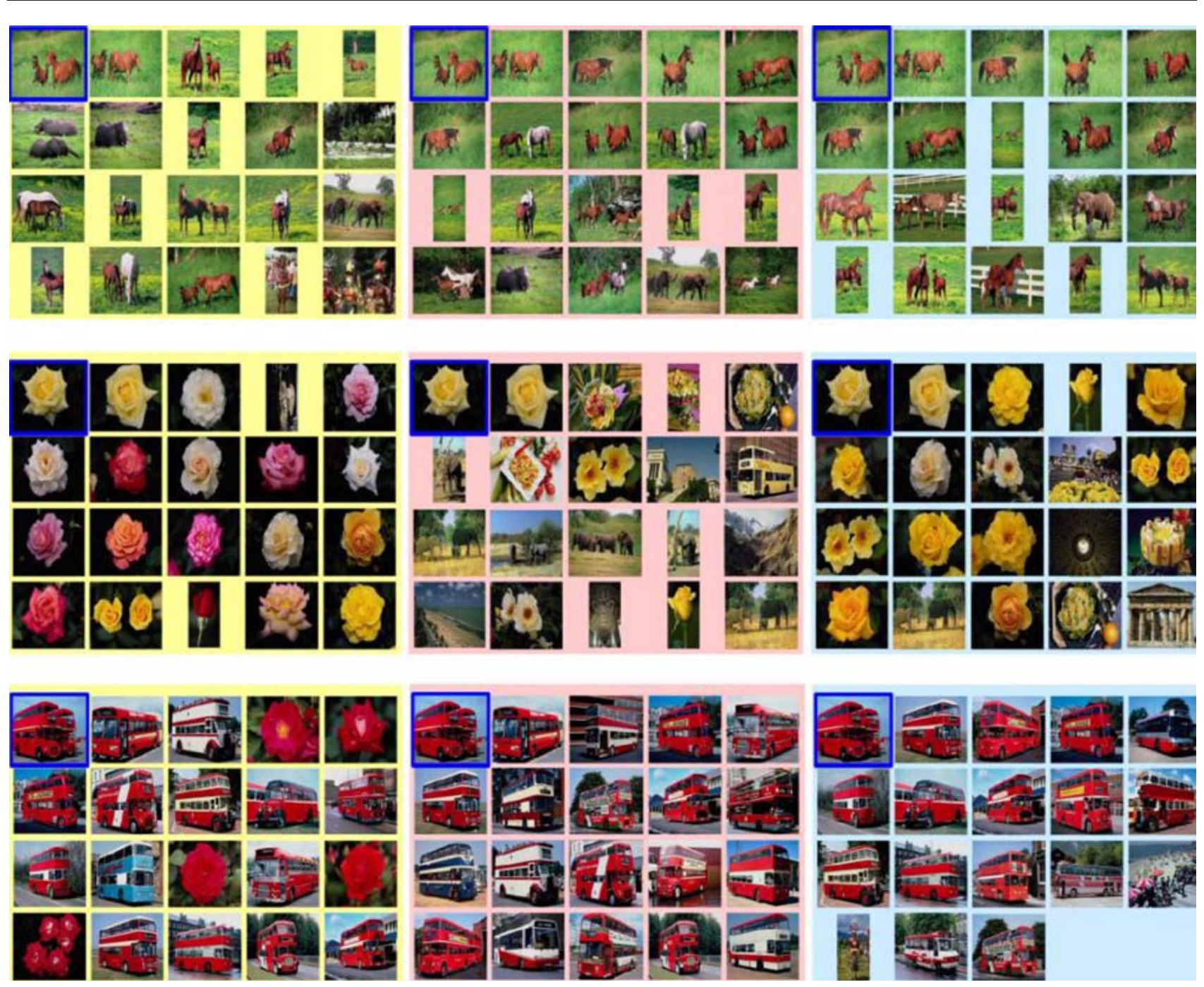

FIG. 4. RETRIEVED IMAGES FOR SAMPLE QUERY IMAGES FROM EACH CATEGORY

\section{CONCLUSION}

The method based on image matching by region based similarity for content based image retrieval is presented. This method depends upon simple but effective metrics that can be described to calculate similarities. Texture and edge features are extracted at region-level whereas shape feature is extracted at image-level. At region-level image is divided in non-overlapping regions. Texture and edge features are calculated for each region separately. Curvelet transform is used for extracting the texture feature by providing the curve continuity as well as line continuity in the feature extraction process. The simulated and measured results based on standard benchmark and exhibit good correspondence. The proposed approach has good performance and produced outstanding results in terms of robust object discovery in mixed class dataset.

\section{ACKNOWLEDGEMENT}

Authors thank reviewers for their valuable comments/ suggestions on the early version of the paper.

\section{REFERENCES}

[1] Memon, M.H., Li, J.P., Memon, I., and Arain, Q. A., "GEO Matching Regions: Multiple Regions of Interests using Content Based Image Retrieval Based on Relative Location", Multimedia Tools and Applications, pp. 1-35, [DOI: 10.1007/s11042-016-3834-z], 2016. 
[2] Memon, I., Chen, L., Majid, A., Lv, M., Hussain, I., and Chen, G., "Travel Recommendation Using Geo-Tagged Photos in Social Media for Tourist”, Wireless Personal Communication, Volume 80, pp. 1347-1362,2015.

Memon, M.H., Li, J-P., Memon, I., Ahmed, R., Shaikh, A.K., and Deep, S., "Unsupervised Feature Approach for Content Based Image Retrieval using Principal Component Analysis”, 11th International Computer Conference on Wavelet Active Media Technology and Information Processing,pp. 271-275,2014.

Song, Y., and Jin, S., "Matching Sequences of Salient Contour Points Characterized by Voronoi Region Features”, Vision Computer, Volume 28, pp. 475-491, [DOI: 10.1007/s00371-011-0643-2], 2012.
Memon, M.H., Li, J-P., Memon, I., Khan, A., Shaikh, R.A., and Deep, S., "Content Based Image Retrieval Based on Geo-Location Driven Image Tagging on the Social Web”, 11th International Computer Conference on Wavelet 937 Active Media Technology and Information Processing, pp. 280-283, 2014.

Memon M.H., Li, J-P., Memon, I., Shaikh, R.A., and Mangi, F.A., "Efficient Object Identification and Multiple Regions of Interest using CBIR Based on Relative Locations and Matching Regions”,12th International Computer Conference on Wavelet Active Media Technology and Information Processing, pp.247-250,[DOI:10.1109/ICCWAMTIP.2015. 7493985], 2015.

Memon, I., and Arain, Q.A., "Dynamic Path Privacy Protection Framework for Continuous Query Service Over Road Networks”, World Wide Web, [DOI: 10.1007/ s11280-016-0403-3], 2017.

Li, J., Qian, X., Li, Q., Zhao, Y., Wang, L., and Tang, Y.Y., “Mining Near Duplicate Image Groups”,Multimed Tools Application, Volume 74, pp. 655-669, [DOI: 10.1007/s11042-014-2008-0], 2015.

Nam, Y., "Real-Time Abandoned and Stolen Object Detection Based on Spatio-Temporal Features in Crowded Scenes”, Multimed Tools Application, Volume75,pp. 7003-7028, [DOI: 10.1007/s11042-0152625-2], 2016.
Belalia, A., Belloulata, K., and Kpalma, K., "RegionBased Image Retrieval in the Compressed Domain using Shape-Adaptive DCT”, Multimed Tools Application, Volume 75, pp. 10175-10199, [DOI: 10.1007/s11042015-3026-2], 2016.

[11] http://wang.ist.psu.edu/jwang/test1.tar (Last Accessed: January, 2013].

[12] Zhang, C., Wang, X., Feng, J., Cheng, Y., and Guo, C., "A Car-Face Region-Based Image Retrieval Method with Attention of SIFT Features”, Multimedia Tools and Applications, Volume 76, No. 8, pp. 10939-10958, 2017.

[13] Niu, S., Chen, Q., de Sisternes, L., Ji, Z., Zhou, Z., and Rubin, D.L. "Robust Noise Region-Based Active Contour Model via Local Similarity Factor for Image Segmentation”, Pattern Recognition, Volume 61, pp. 104-119, 2017.

[14] Zhou, Z., Wang, Y., Wu, Q.J., Yang, C.N., and Sun, X., "Effective and Efficient Global Context Verification for Image Copy Detection”, IEEE Transactions on Information Forensics and Security, Volume 12, No. 1, pp. 48-63, 2017.

[15] Memon, I., “A Secure and Efficient Communication Scheme with Authenticated Key Establishment Protocol for Road Networks”, Wireless Personal Communications, Volume 85, No. 3, pp. 1167-1191, 2015.

[16] Memon, I., Arain, Q.A., Memon, H., and Mangi, F.A., "Efficient User Based Authentication Protocol for Location Based Services Discovery Over Road Networks”, Wireless Personal Communications, pp. 1-20,2017.

[17] Mohammadi, F.G., and Sajedi, H., "Region Based Image Steganalysis using Artificial Bee Colony”, Journal of Visual Communication and Image Representation, Volume 44, pp. 214-226, 2017.

[18] Arain, Q.A., Memon, H., Memon, I., Memon, M.H., Shaikh, R.A., and Mangi, F.A., "Intelligent Travel Information Platform Based on Location Base Services to Predict User Travel Behavior from User-Generated GPS Traces”, International Journal of Computers and Applications, Volume 1, pp. 14, 2017.

[19] http://www.flickr.com 